\title{
Evolution of the dynamical pairing across the phase diagram of a strongly correlated high-temperature superconductor
}

\author{
M. Civelli ${ }^{1}$ \\ 1 Theory Group, Institut Laue Langevin, 6 rue Jules Horowitz 38042 Grenoble Cedex, France
}

(Dated: November 3, 2018)

\begin{abstract}
We study the dynamics of the Cooper pairing across the $T=0$ phase diagram of the twodimensional Hubbard Model, relevant for high-temperature superconductors, using a cluster extension of dynamical mean field theory. We find that the superconducting pairing function evolves from an unconventional form in the over-doped region into a more conventional boson-mediated retarded form in the under-doped region of the phase diagram. The boson, however, promotes the rise of a pseudo-gap in the electron density of states rather than a superconducting gap as in the standard theory of superconductivity. We discuss our results in terms of Mott-related phenomena, and we show that they can be observed in tunneling experiments.
\end{abstract}

PACS numbers: 71.10.-w,71.10.Fd,74.20.-z,74.72.-h

In order to understand high-temperature (high-Tc) superconductivity one must understand the nature of the pairing interaction forming the Cooper pairs. Pairing in standard superconductors has been successfully described by the Migdal-Eliashberg (ME) formalism[1], an extension of the famous Bardeen-Cooper-Schrieffer theory (BCS) of superconductivity [2]. The essential ingredient of the ME description is a retarded boson-mediated interaction between electrons [3]. It has been argued, however, that high-Tc superconductivity is the product of a strongly correlated mechanism [4], which gives rise to an instantaneous electron pairing [5], radically different from the ME description. Recent experimental [6, 7, 8$]$ and theoretical [9, 10, 11] studies on high-Tc superconductors have focused on this problem, but a number of questions remain unanswered. In this paper we enter the debate, examining the pairing interaction as one moves from the over-doped (OD) region to the under-doped (UD) region of the phase diagram of the two-dimensional Hubbard Model (a minimal model containing the physics of high-Tc superconductors [4] ). We look beyond the BCS and ME theories, using the cellular dynamical mean field theory (CDMFT 12, 13]). The CDMFT can address the full frequency-dependence of the pairing interaction and the short-ranged spatial correlation.

It is well established[14] by both experiment[15] and theory [4] (including CDMFT studies 11, 16]) that highTc superconductors have many properties that are BCSlike in the OD region and anomalous in the UD region. Little, however, is known about the dynamics of the Cooper pairing, it being extremely difficult to identify its contribution to physical observables $[\underline{3},[6]$. We show that in the OD region the pairing function does not display BCS or ME forms. Despite the presence of a bosonmediated pairing contribution at low frequencies, other features are relevant up to an energy scale $W^{*}$ of the order of the bandwidth reduced by the strong interaction. In the UD region, however, the pairing function acquires an $\mathrm{ME}$ form, even if at higher energies (but within
$\left.W^{*}\right)$ a pair-breaking contribution appears. We connect these findings with the emergence of a pseudo-gap in the electron spectra at optimal doping (competing with the superconducting gap) and we interpret this in terms of Mott-related phenomena 16, 17]. Finally we show that these properties can be observed in scanning tunneling microscopy (STM) experiments. The two-dimensional Hubbard Model Hamiltonian is:

$$
\mathcal{H}=-\sum_{i, j, \sigma} t_{i j} c_{i \sigma}^{\dagger} c_{j \sigma}+U \sum_{i} n_{i \uparrow} n_{i \downarrow}-\mu \sum_{i \sigma} n_{i \sigma}
$$

where $c_{i \sigma}$ destroys an electron with spin $\sigma$ on site $i$ and $n_{i \sigma}=c_{i \sigma}^{\dagger} c_{i \sigma}$. We only consider the nearest-neighbor $t$ and next-nearest-neighbor $t^{\prime}=-0.3 t$ hoppings; $\mu$ is the chemical potential. We set the on-site Coulomb repulsion $U=12 t$, greater than the bandwidth $8 t$. In CDMFT, $\mathcal{H}$ is mapped onto a more easily handled impurity model $(2 \times 2$ cluster model in our case $)$ of interacting electrons, embedded in a non-interacting bath of fermions, subjected to a self-consistency condition [12]. We solve the cluster-impurity problem using the Lanczos algorithm [18], which approximates the non-interacting bath with an 8-level parameterization. This method works at zero temperature [19] and provides direct access to the information on the real-frequency axis, unlike, for example, the quantum-Monte-Carlo method(QMC) 20].

In this study we consider a paramagnetic translationally invariant superconducting state by constraining the CDMFT equations. Even if other broken-symmetry phases compete for the ground-state (at low doping, antiferromagnetism is expected to take over, for example), this is a well-defined mean-field procedure which allows us to access and study the physics governing the superconductivity. We leave open the question of which terms could be added to $\mathcal{H}$ to make this superconducting mean-field solution into a real groundstate. The output of our CDMFT calculation is the frequency-dependent normal $G_{i j}^{n o r}(\omega)=\ll c_{i \sigma} c_{j \sigma}^{\dagger} \gg$ and anomalous $F_{i j}(\omega)=\ll c_{i \uparrow} c_{j \downarrow} \gg$ cluster Green's func- 


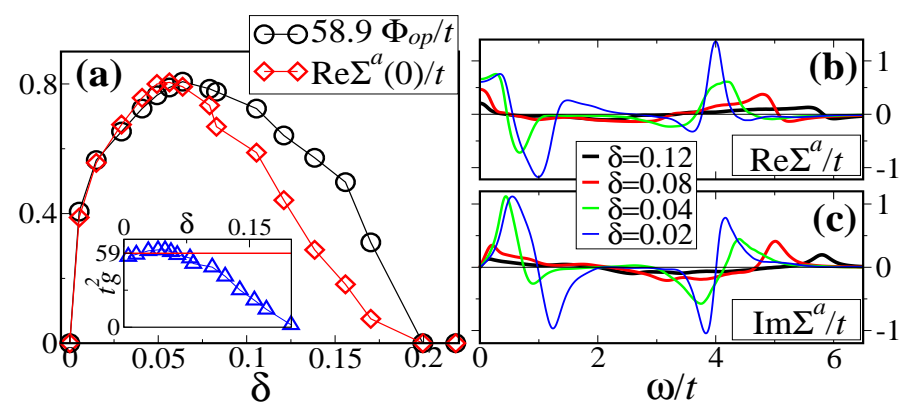

FIG. 1: (color online). The superconducting order parameter $\Phi_{o p}$ (multiplied by $t^{2} g=58.9$ shown in the inset) and the anomalous self-energy value $\operatorname{Re} \Sigma^{a}(\omega=0)$ are displayed as a function of doping $\delta$ (a). The $\operatorname{Re} \Sigma^{a}(\omega)$ (b) and $\operatorname{Im} \Sigma^{a}(\omega)$ (c) are displayed on a wide energy range $0<\omega<U / 2$, from the OD region $(\delta=0.12,0.08)$ to the UD region $(\delta=0.04,0.02)$.

tions and their associated normal $\sum_{i j}^{n o r}(\omega)$ and anomalous $\sum_{i j}^{a n o}(\omega)$ cluster self-energies $(i, j=1, \ldots, 4$ in the $2 \times 2$ cluster [16, 21]). We indicate the Fourier transform of the time-ordered ground-state average with $\ll \ldots \gg$. In this paper we focus on the nearest-neighbor anomalous component $\Sigma^{a}(\omega)$ (the only one numerically nonzero), which directly expresses the pairing function. We think that it captures the essential features of the superconducting pairing, which has a d-wave momentum dependence $\sim\left(\cos k_{x}-\cos k_{y}\right)$ (dominated by the nearestneighbor spatial component 22]), as has been well established experimentally [15] and theoretically [14, 23].

In Fig. 17 the superconducting order parameter $\Phi_{o p} \equiv$ $\int_{-\infty}^{\infty} F_{12}(\omega) d \omega$ is displayed as a function of doping $\delta=$ $1-\left\langle n_{i}\right\rangle . \Phi_{o p}$ has the expected dome-like shape, with a maximum around $\delta_{o p t} \sim 0.07$; this locates the optimal doping in our Lanczos-CDMFT calculation (a similar $\delta_{\text {opt }}$ is also obtained in the QMC-CDMFT results of Ref.[11]). This value is smaller than the $\delta_{\text {opt }} \sim 0.15$ observed in cuprate materials, which require more complete models to be described in detail. However, as with the cuprates, we shall define the region of $\delta>\delta_{\text {opt }}\left[\delta<\delta_{\text {opt }}\right]$ as the OD region [UD region]. If we compare $\Phi_{o p}$ with the anomalous self-energy value $\Sigma^{a}(\omega=0)$ we can already observe the unusual non-BCS behavior of the Cooper pairing in the OD region. If BCS theory is a good approximation, $\Sigma^{a}(0) \sim g \Phi_{o p}$, where $g$ is the strength of the pairing interaction 24]. But $\Sigma^{a}(0)$ roughly scales like $\Phi_{o p}$ only in the UD region (with $g \sim 58.9$ ), with no scaling possible in the OD region (as is shown by the $\delta$-dependence of $g$ in the inset).

To clarify the observation above we investigated the $\omega$-dependence of $\Sigma^{a}(\omega)$ (Fig. 1 b,c). As with previous results[9, 11], $\Sigma^{a}(\omega)$ shows features which extend to high energies $(\omega \sim 6 t) . \operatorname{Re} \Sigma^{a}$ assumes negative values; these are particularly evident in the UD region (around $0.5 t<$ $\omega<3.5 t$ ), indicating a repulsive pair interaction. In
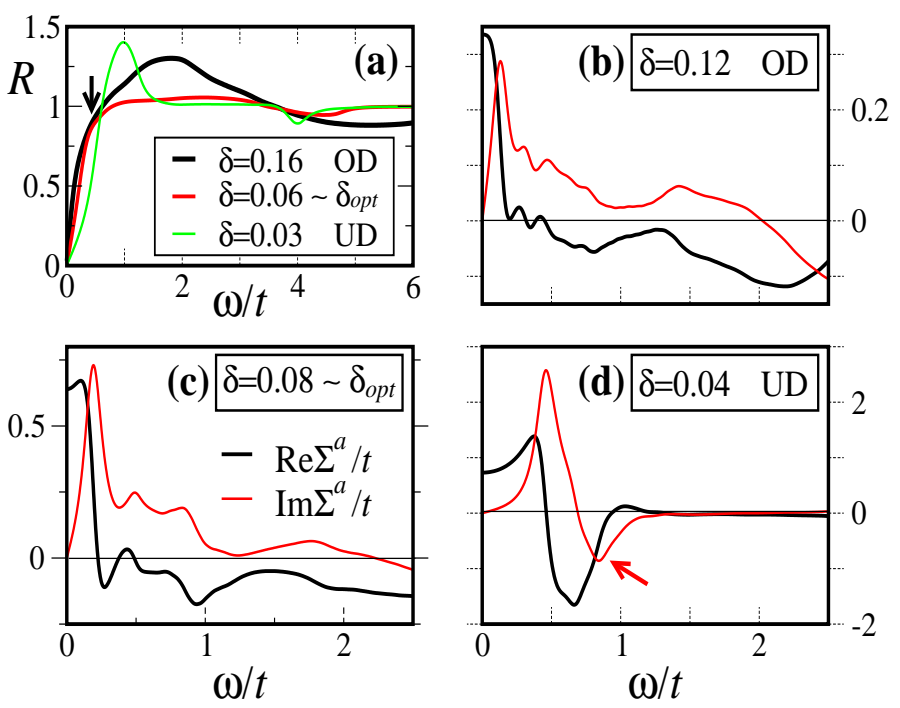

FIG. 2: (color online). The ratio $R$ (defined in formula 2) vs. $\omega$ (a). The low-energy close-up $\left(\omega<W^{*}\right)$ of $\operatorname{Re} \Sigma^{a}(\omega)$ and $\operatorname{Im} \Sigma^{a}(\omega)$ is displayed in the OD region (b), close to optimal doping $\delta_{o p t}(\mathrm{c})$ and in the UD region $(\mathrm{d})$.

absolute value $\Sigma^{a}$ decreases with doping, although Fig. 13 shows that $\operatorname{Re} \Sigma^{a}(\omega \rightarrow 0)$ increases in the UD region (in agreement with the QMC-CDMFT results[11]).

We now look in detail at the evolution of $\Sigma^{a}$ across the phase diagram. We first employ Cauchy-KramersKroenig relations and define the ratio 9 , 22]

$$
R(\omega)=\frac{1}{\pi} \frac{1}{\operatorname{Re} \Sigma^{a}(0)} \int_{0}^{\omega} d \nu \frac{\operatorname{Im} \Sigma^{a}(\nu)}{\nu}
$$

$R(\omega)$ measures the contribution to the low-energy superconducting pairing $\operatorname{Re} \Sigma^{a}(\omega=0)$ obtained from the range of frequency up to $\omega$. We identify three distinct doping-dependent regimes (Fig. 2a). (i) In the OD region, $R(\omega)$ monotonically increases to $\omega \sim W^{*} \sim 2 t$, and then decreases, showing a wide hump which overshoots one, centered around $\omega \sim 1.5 t$. The small hump also visible (small arrow) around $\omega \sim 0.5 t$ indicates the presence of a boson-like contribution to $\Sigma^{a}$. (ii) Close to optimal doping $\delta_{o p t}$, the range of increasing monotonicity of $R(\omega)$ is reduced to $\omega \leq t$. For $\omega>t, R(\omega)$ stays roughly flat around one. (iii) In the UD region, a narrow peak overshooting one appears at low frequency $\omega \leq t$. This form of $R(\omega)$ is similar to that expected from a boson-mediated pairing mechanism [3, 9].

$R(\omega)$ shows that the pairing builds up for $\omega \leq$ $W^{*}$ and hints at the presence of a low-energy boson. Bosonic modes have actually been detected in high-Tc materials $[6,7,6]$. Figs. 2 b,c,d, which show the low energy part of $\Sigma^{a}$, therefore merit close examination. In ME theory, the boson exchange between pairing electrons resonates as $\omega \rightarrow \omega_{o}^{-}$, a characteristic frequency. This fact is made evident by a hump in $\operatorname{Re} \Sigma^{a}(\omega)$. For 

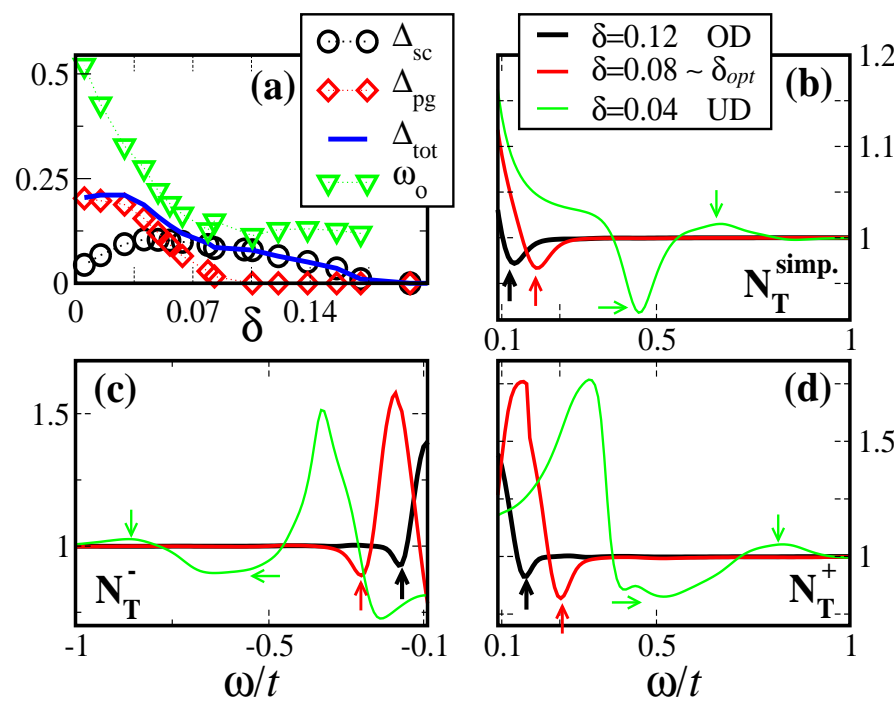

FIG. 3: (color online). The superconducting gap $\Delta_{s c}$, the pseudo-gap $\Delta_{p g}, \Delta_{t o t}=\sqrt{\Delta_{s c}^{2}+\Delta_{p g}^{2}}$ and the characteristic boson-frequency $\omega_{o}$ are displayed as a function of doping $\delta$ (a). The conductance ratio $N_{T}^{\text {simp. }}$ (b) is a simple ideal case (Eq. 3), where only the $\Sigma^{a}$-dependence is relevant. $N_{T}^{-}$(c) and $N_{T}^{+}$(d) represent more realistic-material case, obtained via a periodization [16] in momentum space of the CDMFT results.

$\omega \geq \omega_{o}$ however, the probability of emission of a real boson increases; this is marked by $\operatorname{Re} \Sigma^{a}(\omega)$ changing sign and by $\operatorname{Im} \Sigma^{a}(\omega)$ acquiring a peak. This peak occurs at $\Omega \sim \omega_{o}+\operatorname{Re} \Delta_{t o t}(0), \operatorname{Re} \Delta_{t o t}(0)$ being the lowest particleexcitation available in the superconductor (the superconducting gap in a standard BCS superconductor). In the OD region (Fig. 2b), $\Sigma^{a}$ has the ME form described above at low $\omega$, but it also has other features at higher frequencies. The pair interaction is repulsive for $\omega \geq 0.5 t$, where $\operatorname{Re} \Sigma^{a}$ changes sign. The $\operatorname{Im} \Sigma^{a}$ however has a long positive tail until $\omega \sim W^{*} \sim 2 t$, which means that the pairing mechanism involves states in the renormalized band of width $W^{*}$. The superconducting pairing in the OD region cannot therefore be described simply within the ME theory, which typically shows only one or few characteristic boson-frequencies (where $\operatorname{Im} \Sigma^{a}$ shows well defined peaks). Around $\delta_{\text {opt }}$ (Fig. 2r) the long tail in $\operatorname{Im} \Sigma^{a}$ reduces strongly. In the UD region (Fig. $2 \mathrm{~d}$ ), the tail disappears, and $\Sigma_{\text {ano }}$ assumes an ME shape (in line with the findings of Fig. 1), marking a change in the pairing mechanism.

We now link the evolution of the pairing function with the relevant energy-scales of the system. In BCS theory there is only one such scale, the superconducting gap $\Delta_{s c} \sim \omega_{o} \mathrm{e}^{-1 /\left(N_{o} g\right)}$ (where $N_{o}$ is the density of states at the Fermi level), proportional to the characteristic boson-frequency $\omega_{o}$. In our results, however, there are two relevant energy-scales [16, 17]. The first de- rives from $\operatorname{Re} \Sigma^{a}$ and represents the superconducting gap $\Delta_{s c} \sim Z_{k_{n o d}} \operatorname{Re} \Sigma^{a}(0)$ (where $Z_{k_{n o d}}$ is the quasiparticle residue [16, 17]). In our theory, $\Delta_{s c}$ can be measured in the regions of momentum space close to "nodal" points $\mathbf{k}_{\text {nod }} \sim\left( \pm \frac{\pi}{2}, \pm \frac{\pi}{2}\right)$ by photo-emission [25, 26]; its behavior is non-monotonic with doping (Fig. 3a). The second energy scale $\Delta_{p g}$ arises in the UD region from the normal component $\Sigma^{\text {nor }}$ (see Ref.[16, 17, 27]); it is connected with the pseudo-gap observed in the normal state, and it is monotonic with $\delta$. It is convenient to define a total gap [16] $\Delta_{t o t}=\left(\Delta_{p g}^{2}+\Delta_{s c}^{2}\right)^{\frac{1}{2}}$, which is measured in photoemission [25, 26] close to the "antinodal" points $\mathbf{k}_{\text {anod }} \sim(0, \pm \pi)$ or $( \pm \pi, 0)$. In the OD region $\Delta_{s c} \equiv \Delta_{\text {tot }}$, but in the UD region the contribution of $\Delta_{p g}$ at the antinodes is most important [16, 17]. To clarify whether the boson-feature observed in Fig. 2 can be related to any of these gaps, we estimate its characteristic frequency $\omega_{o} \sim \Omega-\Delta_{\text {tot }}\left(k_{\text {anod }}\right)$ (at $\omega=\Omega$ we locate the first maximum of $\operatorname{Im} \Sigma^{a}(\omega)$ in Fig. 2 $\left.2 \mathrm{~b}, \mathrm{c}, \mathrm{d}\right)$ and plot it as a function of doping $\delta$. We find that $\omega_{o}$ follows the behavior of the pseudo-gap $\Delta_{p g}$ (suggestive of the experimental results in Ref. [6]) rather than the superconducting gap $\Delta_{s c}$. Coming from the OD region, $\omega_{o}$ is roughly constant, just as $\Delta_{p g}=0$, while $\Delta_{s c}$ increases. In the UD region, $\omega_{o}$ monotonically increases as doping is reduced; it follows the rise of $\Delta_{p g}$ rather than the fall of $\Delta_{s c}$, in spite of the ME form of $\Sigma^{a}$. This is due to the fact that, in the UD region, negative values appear in $\operatorname{Im} \Sigma^{a}$ (oblique arrow in Fig. $2 \mathrm{~d}$ ) which give a negative contribution to the $\operatorname{Re} \Sigma^{a}(0)$ (Eq. 2) and break the Cooper pairs.

In our interpretation [16, 17, 27], these findings are Mott-related phenomena. At optimal doping $\delta_{o p t}$ the antinodal regions of momentum space undergo a Mott transition $\left(\Delta_{p g} \geq 0\right)$, while the nodal regions retain a metallic character. These ideas [11, 28] are reminiscent of the orbitally selective Mott transition in multi-band models. Close to the Mott transition, antiferromagnetic fluctuations are important [9, 29, 30], and can mediate a pairing channel $\left\langle c_{i \uparrow} c_{j \downarrow}\right\rangle$ to form Cooper pairs $[9]$. The proximity to the "antinodal" Mott transition results in a pairing process involving electrons on a wide energy scale (with $\operatorname{Im} \Sigma^{a}$ positive up to $\omega \sim W^{*}$ ). The highTc mechanism therefore originates as one approaches a Mott transition [4]. Once this latter has taken place at $\delta_{\text {opt }}$, a normal-component particle-hole $\left\langle c_{i \sigma}^{\dagger} c_{j \sigma}\right\rangle$ channel is opened, destroying the Cooper pairing at higher energies $\left(\Delta_{s c}\right.$ decreases) and favoring the rise of $\Delta_{p g}$. This is marked by the appearance of non-BCS properties in the normal component together with a pairing function of a more standard ME form.

Finally, let us look at how the doping-dependent description of the pairing function can be measured in experiments. In ME superconductivity it was possible to relate the behavior of the superconducting pairing to STM measurements [3] of the ratio $N_{T}$, between the superconducting and normal-state tunneling conductances 
$d I(\omega) / d V$, obtained under the same conditions:

$$
\begin{aligned}
N_{T}(\omega) & =\frac{d I(\omega) /\left.d V\right|_{s c}}{d I(\omega) /\left.d V\right|_{n o r}}=\frac{N_{s c}(\omega)}{N_{n o r}(\omega)} \approx \\
N_{T}^{\text {simp. }}(\omega) & =1+\frac{Z_{k_{n o d}}}{4 \omega^{2}}\left[\operatorname{Re} \Sigma^{a}(\omega)^{2}-\operatorname{Im} \Sigma^{a}(\omega)^{2}\right]
\end{aligned}
$$

where $N_{s c[n o r]}(\omega)=\frac{1}{\pi} \sum_{k} \operatorname{Im} G(k, \omega)$ is the superconducting [normal-state] local density of states. $N_{T}^{\text {simp. }}$ is the ideal $\omega \rightarrow \infty$ limit, obtained in the simplest ME analysis of a d-wave superconductor [3, 6] neglecting the momentum dependence of the band-structure and taking $N_{\text {nor }}(\omega) \approx N_{\text {nor }}(0)$ (a good approximation

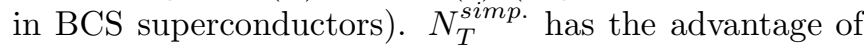
being an explicit function of the superconducting gap $\Delta_{s c}(\omega) \sim Z_{k_{\text {nod }}} \Sigma^{a}(\omega)$. Its behavior is shown in Fig. 3b. The sharp drop below unity (due to the $\operatorname{Re} \Sigma^{a} \rightarrow 0$ and $\operatorname{Im} \Sigma^{a}$ acquiring a maximum, see Fig. 2) marks the presence of the boson (at $\omega=\Omega$, vertical-up and horizontal arrows in Fig. [3b), both in the OD region (in agreement with experiments[6]) and in the UD region. It is not possible to see the long tail of the $\operatorname{Im} \Sigma^{a}$ in the OD region, as the real and imaginary parts of $\Sigma^{a}$ cancel out. We can however observe two clear features: (i) the sudden increase of $\Omega$ in passing from the OD region to the UD region, mainly due to the increase of $\omega_{o}$, as portrayed in Fig. 3 a , and (ii) the rise above unity at higher frequencies (marked by a vertical-down arrow) in the UD region; this is due to the pair-breaking effect of $\operatorname{Im} \Sigma^{a}$ assuming negative values (in a way opposite to the boson-drop, now $\operatorname{Im} \Sigma^{a} \rightarrow 0$, and $\operatorname{Re} \Sigma^{a}$ is maximal). The approximations used to extract the simple behavior of $N_{T}^{\text {simp }}$ no longer hold in the real-case situation, where the momentum dependence of the band cannot be neglected and, above all, $N_{\text {nor }}(\omega)$ is strongly $\omega$-dependent. We performed a full momentum-energy-dependent calculation, however, adopting a mixed-periodization scheme (introduced in Ref.[16, 17]), which allows us to extract a good approximation for $G(k, \omega)$ from the cluster results, while separating the normal component $N_{\text {nor }}$ from the superconducting $N_{s c}$. The resulting $N_{T}^{+}$for $\omega>0$ and $N_{T}^{-}$for $\omega<0$ are displayed in Figs. 3r,d. The curves are now more irregular and asymmetric with respect to $\omega=0$, but the features (i) and (ii) which we have discussed for $N_{T}^{\text {simp }}$ are still present, showing that it should be possible to observe them in experiments [6] .

We can conclude that the experiments and theories showing unconventional properties in the UD region and more conventional properties in the OD region of the phase diagram of high-Tc superconductors do not tell the entire story. We find an unconventional form of superconducting pairing in the OD region, which evolves into a more standard form in the UD region. This crossover takes place close to the maximum of the order parameter, where a pseudo-gap appears in the one-electron density of states. We argue that these phenomena are a con- sequence of a Mott-like transition taking place at the antinodal points of momentum space.

We would like to thank G. Kotliar, B. Kyung, Th. Maier, I. Paul, D. Poilblanc, A.-M. Tremblay, R.S. Whitney for the helpful discussions and P. Bruno, A. Cano, C. Pepin, T. Ziman for their comments. We also thank R. Corner for proofreading this article.

[1] A.B. Migdal, Zh. Eksp. Teor. Fiz., 34 (1958) 1438. (Sov. Phys. JETP, 7 (1958) 996); G.M. Eliashberg, Zh. Eksp. Teor. Fiz., 38 (1960) 966; 39 (1960) 1437. (Sov. Phys. JETP, 11 (1960) 696; 12 (1960) 1000).

[2] J. Bardeen, L.N. Cooper and J.R. Schrieffer, Phys. Rev. 106, 162 (1957).

[3] See e.g.: D.J. Scalapino, in Superconductivity, edited by R.D. Parks (Marcel Dekker) 1969, p. 449.

[4] P.W. Anderson, Science 235, 1196 (1987).

[5] P.W. Anderson, Science 316, 1705 (2007).

[6] A.N. Pasupathy et al., Science 320, 196 (2008).

[7] E. van Heumen et al., arXiv:0807.1730.

[8] E.G. Maksimov, M.L. Kulié and O.V. Dolgov, arXiv:0810.3789.

[9] T.A. Maier, D. Poilblanc and D.J. Scalapino, Phys. Rev. Lett. 100, 237001 (2008).

[10] R.S. Markiewicz and A. Bansil, Phys. Rev. B 78, 134513 (2008).

[11] K. Haule and G. Kotliar, Phys. Rev. B 76, 104509 (2007).

[12] G. Kotliar et al., Rev. of Mod. Phys. 78, 000865 (2006).

[13] A.-M.S. Tremblay, B. Kyung and D. Sénéchal, Low Temp. Phys., 32, 561-595 (2006); Th. Maier et al., Rev. of Mod. Phys. 77, 1027-1080 (2005).

[14] "Physics of Superconductors II", K.H. Bennemann and J.B. Ketterson, Springer-Verlag Berlin (2004).

[15] A. Damascelli, Z.X. Shen and Z. Hussain, Rev. Mod. Phys. 75, 473 (2003); J.C. Campuzano, M. R Norman and M. Randeria, pg. 167-273, in [14].

[16] M. Civelli, Phys. Rev. B 79, 195113 (2009).

[17] M. Civelli et al., Phys. Rev. Lett. 100, 046402 (2008).

[18] M. Caffarel and W. Krauth, Phys. Rev. Lett. 72, 1545 (1994).

[19] Implementation requires introducing a distance function and a low energy cutoff, set by $\beta t=300$, on the imaginary axis $\omega_{n}=(2 n-1) \pi / \beta$ (for details see [12, 16]).

[20] J.E. Hirsch and R.M. Fye, Phys. Rev. Lett. 56, 2521 (1986); P. Werner et al., Phys. Rev. Lett. 97, 076405 (2006).

[21] S.S. Kancharla et al., Phys. Rev. B 77, 184516 (2008).

[22] D. Poilblanc and D.J. Scalapino, Phys. Rev. B 71, 174403 (2005); D. Poilblanc, D.J. Scalapino and S. Capponi, Phys. Rev. Lett. 91, 137203 (2003).

[23] G. Kotliar and J. Liu, Phys. Rev. B 38, 5142 (1988).

[24] See e.g. chapter 51 in: A.L. Fetter and J.D. Walecka, Quantum Theory of Many-Particle Systems, Dover Publications, Mineola NY, 2003.

[25] M. Le Tacon et al., Natur. Phys. 2, 537 (2006); W. Guyard et al., Phys. Rev. Lett. 101, 097003 (2008).

[26] K. Tanaka et al., Science 314, 1910 (2006).

[27] T.D. Stanescu and G. Kotliar, Phys. Rev. B 74, 125110 (2006). 
[28] M. Ferrero et al., Europhys. Lett. 85, 57009 (2009).

[30] S. Brehm et al., arXiv:0811.0552.

[29] Hyungje Woo et al., Natur. Phys. 2, 600 (2006). 